\title{
Integer quantum Hall effect in gapped single-layer graphene
}

\author{
P. M. Krstajić ${ }^{1}$ and P. Vasilopoulos ${ }^{2}$ \\ ${ }^{1}$ Institute of Microelectronic Technologies and Single Crystals (IHTM), University of Belgrade, Njegoševa 12, 11000 Belgrade, Serbia \\ ${ }^{2}$ Concordia University, Department of Physics, 7141 Sherbrooke Ouest, Montréal, Québec, Canada H4B 1R6
}

(Received 26 June 2012; published 20 September 2012)

\begin{abstract}
Analytical expressions for the Hall conductivity $\sigma_{y x}$ and the longitudinal resistivity $\rho_{x x}$ are derived in gapped, single-layer graphene using linear response theory. The gap $2 \Delta$, described by a mass term, is induced by a substrate made of hexagonal boron nitride (h-BN) and produces two levels at $\pm \Delta$. It is shown that $\sigma_{y x}$ has the same form as for a graphene sample supported by a common substrate without a mass term. The differences are a shift in the energy spectrum, which is not symmetric with respect to the Dirac point for either valley due to the gap, the absence of a zero-energy Landau level, and the nonequivalence of the $K$ and $K^{\prime}$ valleys. In addition, the dispersion of the energy levels, caused by electron scattering by impurities, modifies mostly plateaus due to the levels at $\pm \Delta$. It is shown that the resistivity $\rho_{x x}$ exhibits an oscillatory dependence on the electron concentration. The main difference with the usual graphene samples, on $\mathrm{SiO}_{2}$ substrates, occurs near zero concentration, as the energy spectra differ mostly near the Dirac point.
\end{abstract}

DOI: $10.1103 /$ PhysRevB.86.115432

PACS number(s): 81.05.ue, 72.80.Vp, 73.43.-f

\section{INTRODUCTION}

There has been increased interest in graphene single layers on substrate made of hexagonal boron nitride (h-BN). ${ }^{1-6}$ This substrate has its own advantages over $\mathrm{SiO}_{2}$, since it has a smooth surface on the atomic scale and its lattice constant is close to that of graphene. Also, it is relatively free of dangling bonds and charge traps. ${ }^{1}$ Furthermore, graphene devices on h-BN substrates exhibit higher mobilities ${ }^{1,2}$ and reduced carrier inhomogeneities and roughness. ${ }^{1}$ The novel substrate induces a finite gap and brings about nonequivalence of the two sublattices that make up the honeycomb crystal structure of graphene. The gap is on the order of several tens of meV at the Dirac point and leads to finite masses for the Dirac fermions. So far, though, this gap has not been determined experimentally in an unambiguous way. There are indications of its existence but one is left with a theoretical value determined from $a b$ initio calculations. ${ }^{3}$ An alternative way to introduce a gap in graphene monolayer using h-BN is to synthesize atomically thin films composed of hybridized h-BN and graphene domains as reported in Ref. 7. This novel material exhibits structural properties and a band gap that have not been found in graphene, h-BN, or boron- and nitrogen-codoped graphene. In addition, this new structure may be utilized to make unique semiconducting 2D architectures, which could find applications in electronics.

As far as related theoretical papers are concerned, we first mention a study of the valley Hall effect ${ }^{8}$ in which the authors found an analytical expression for the Hall conductivity as a function of the valley index and a gap between the two sublattices of graphene in the absence of an external magnetic field. Further, zigzag-terminated graphene nanoribbons placed on a h-BN substrate have been investigated ${ }^{9}$ and predicted to behave like perfect spin filters or analyzers. A theoretical paper whose subject is closest to ours appeared recently ${ }^{5}$ and deals with magnetotransport properties of a gapped monolayer graphene. More precisely, the authors of Ref. 5 used the Kubo formula and the self-consistent Born approximation in order to calculate the Hall conductivity and the longitudinal conductivity in the presence of long-range impurity potentials.
Their results suggest that the anomalous integer quantum Hall effect (IQHE) is preserved under the condition the mass term is not too large compared to the cyclotron energy. Though that approach is correct, it is a purely numerical one. In this paper we follow an alternative approach that employs simpler linear response formulas and yields clear and analytical expressions with which it is possible to gain more physical insight into the problem.

The paper is organized as follows. The theoretical model of magnetotransport properties of graphene on a h-BN substrate is presented in Sec. II as well as the expressions for the Hall conductivity $\sigma_{y x}$ and the longitudinal conductivity $\sigma_{x x}$. Various numerical results for $\sigma_{y x}$ and the resistivity $\rho_{x x}$ are presented and discussed in Sec. III, and Sec. IV summarizes the main results.

\section{THEORETICAL MODEL}

When a graphene sample is placed on a boron-nitride substrate a staggered potential is formed in the plane of graphene. In practice, this means that the on-site energies of the carbon atoms belonging to the $A$ and $B$ sublattices have different values, denoted by $\Delta$ and $-\Delta$, respectively. As a consequence, an energy gap between the conduction and valence bands appears. A new situation brought about by the staggered potential can still be described by a Dirac-like Hamiltonian $^{5}$ but with additional diagonal terms. More precisely, the Hamiltonian of gapped graphene in a perpendicular magnetic field $B$ in the vicinity of the $K$ point is given by ${ }^{5}$

$$
H=\left(\begin{array}{cc}
\Delta & v_{F} p_{-} \\
v_{F} p_{+} & -\Delta
\end{array}\right),
$$

where $v_{F}$ denotes the Fermi velocity, $\mu_{B}$ is the Bohr magneton, and $p_{ \pm}=p_{x} \pm i p_{y}$. The Hamiltonian in Eq. (1) is written in $(2+1)$ dimensions. In the presence of a magnetic field one uses the Pierls substitution $\mathbf{p} \rightarrow \mathbf{p}+e \mathbf{A}(e>0)$. The Zeeman term in Eq. (1) is neglected since it is important only at very high magnetic fields $(B>20 \mathrm{~T}) .^{10-12}$ The energy spectrum is then expressed in terms of the cyclotron energy $\hbar \omega_{c}=\hbar \sqrt{2} v_{F} / \ell_{c}$, 
where $\ell_{c}=\sqrt{\hbar / e B}$ is the magnetic length. Furthermore, by choosing the Landau gauge $\mathbf{A}=(-B y, 0)$ and making the substitutions $\pi_{ \pm}=p_{x} \pm i p_{y}-e B y$, one readily arrives at

$$
H=\left(\begin{array}{cc}
\Delta & v_{F} \pi_{-} \\
v_{F} \pi_{+} & -\Delta
\end{array}\right) .
$$

The normalized eigenvectors of the eigenvalue problem $H \Psi=$ $E \Psi$ are

$$
\Psi_{n}=\frac{1}{\sqrt{L_{x}}}\left(\begin{array}{c}
a_{s n} \Phi_{n-1} \\
s b_{s n} \Phi_{n}
\end{array}\right) e^{i k_{x} x},
$$

where $\Phi_{n}(\xi)$ are the usual oscillator functions with $\xi=$ $\left(y-\ell_{c}^{2} k_{x}\right) / \ell_{c}$. The index $n(n=0,1,2, \ldots)$ labels the discrete Landau levels while $s$ labels the conduction $(s=+1)$ and valence bands $(s=-1)$. With $E_{s n}$ being the eigenvalues the coefficients $a_{s n}$ and $b_{s n}$ are given by

$$
a_{s n}=\sqrt{\frac{E_{s n}+\Delta}{2 E_{s n}}}, \quad b_{s n}=\sqrt{\frac{E_{s n}-\Delta}{2 E_{s n}}} .
$$

The case of the zeroth level $\left|s, 0, k_{x}\right\rangle$ should be treated separately as usual. In gapless graphene $(\Delta=0)$, this zeroenergy level is placed at the Dirac point and is equally shared between the conduction and valence bands. This has the consequence that its degeneracy is twice as small as that of other levels (when both valleys are taken into account). Its normalized eigenvector has the form

$$
\Psi_{0}=\frac{1}{\sqrt{L_{x}}}\left(\begin{array}{c}
0 \\
\Phi_{0}
\end{array}\right) e^{i k_{x} x} .
$$

Notice that $\left|+, 0, k_{x}\right\rangle$ and $\left|-, 0, k_{x}\right\rangle$ correspond to the same level. For $n \geqslant 0$ the eigenvalues of Eq. (2) are given by

$$
E_{s n}=s\left[\Delta^{2}+n \hbar^{2} \omega_{c}^{2}\right]^{1 / 2}\left(1-\delta_{n 0}\right), \quad E_{s 0}=\mp \Delta \delta_{n 0} .
$$

The sign of the zeroth level energy $E_{s 0}$, is negative for the $K$ point and positive for the $K^{\prime}$ point. Later, we will introduce the valley index $v=1,-1$, which is not an additional quantum number as there are two independent $(2 \times 2)$ Hamiltonians (one for each valley). Figure 1 shows the energy spectrum of single graphene layer in the presence of perpendicular magnetic field $B=14 \mathrm{~T}$ for both valleys (denoted by red lines online). The spectrum of graphene in the absence of the magnetic field $(B=0 \mathrm{~T})$ is given, versus $k \ell_{c}$, by the black solid
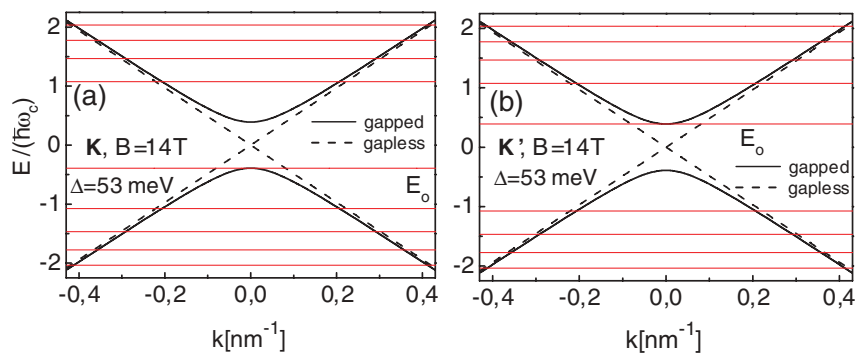

FIG. 1. (Color online) Energy spectrum of gapped graphene (horizontal lines) in the presence of a magnetic field for the $K$ valley in (a) and the $K^{\prime}$ one in (b). The conical-like energy dispersion gives the spectrum (solid lines) versus $k$ for $\Delta \neq 0$ and the dashed lines that of gapless graphene for $B=0$. curves, while the dashed lines show that of gapless graphene. Note that, due to the mass term $\Delta$, cf. Eq. (6), the spectrum versus $k$ is not symmetric with respect to the Dirac point.

Assuming that the electric, source-drain field is weak, one may use linear response theory ${ }^{13}$ and obtain the Hall conductivity in the form ${ }^{12}$

$$
\sigma_{\mu \nu}^{n d}=\frac{i \hbar e^{2}}{S_{0}} \sum_{\zeta \neq \zeta^{\prime}} \frac{\left(f_{\zeta}-f_{\zeta^{\prime}}\right) v_{v \zeta \zeta^{\prime}} v_{\mu \zeta^{\prime} \zeta}}{\left(E_{\zeta}-E_{\zeta^{\prime}}\right)\left(E_{\zeta}-E_{\zeta^{\prime}}+i \Gamma_{\zeta}\right)},
$$

where $S_{0}$ is the area and $v_{\nu, \mu \zeta \zeta^{\prime}}$ the matrix elements of the velocity operator, $\mu, v=x, y$. Further, $f_{\zeta}=[1+$ $\left.\exp \beta\left(E_{\zeta}-E_{F}\right)\right]^{-1}$ is the Fermi-Dirac distribution function with $\beta=1 / k_{B} T$, where $T$ the temperature and $E_{F}$ is the Fermi level.

The sum runs over all quantum numbers $|\zeta\rangle=\left|s, n, k_{x}\right\rangle$ and $\left|\zeta^{\prime}\right\rangle=\left|s^{\prime}, n^{\prime}, k_{x}^{\prime}\right\rangle$ provided $\zeta \neq \zeta^{\prime}$. The infinitesimal quantity $\epsilon$ in the original form ${ }^{13}$ has been replaced by $\Gamma_{\zeta}$ to account for the finite broadening of the Landau levels. Assuming that this broadening is approximately the same for all states ${ }^{14} \Gamma_{\zeta} \approx \Gamma$, one can demonstrate that the imaginary part of Eq. (7) vanishes. Further, it was proven ${ }^{14,15}$ that $\Gamma$ increases with the magnetic field like $\propto \sqrt{B}$ or, more precisely, ${ }^{14} \Gamma=\sqrt{2 / A} \hbar \omega_{c}$. Here the dimensionless parameter $A$ depends on the concentration of scattering centers and has values in the range 50-100. In the spirit of the original derivation of Eq. (7), and in order to obtain a transparent result for $\sigma_{y x}$, we take $\Gamma=0$. We will consider a finite $\Gamma$ later in the evaluation of the longitudinal conductivity.

To proceed, one needs the product of the velocity matrix elements for $v=x$ and $\mu=y$ (diagonal in $k_{x}$ )

$$
P_{n n^{\prime}}^{s s^{\prime}}=\left\langle s n k_{x}\left|v_{x}\right| s^{\prime} n^{\prime} k_{x}^{\prime}\right\rangle\left\langle s^{\prime} n^{\prime} k_{x}^{\prime}\left|v_{y}\right| s n k_{x}\right\rangle .
$$

To simplify the notation used, henceforth the quantum number $k_{x}$ is suppressed. After an explicit evaluation of the product $P_{n n^{\prime}}^{s s^{\prime}}$ one obtains

$$
P_{n n^{\prime}}^{s s^{\prime}}=i v_{F}^{2}\left(\left|a_{s n} b_{s^{\prime} n^{\prime}}\right|^{2} \delta_{n-1, n^{\prime}}-\left|a_{s^{\prime} n^{\prime}} b_{s n}\right|^{2} \delta_{n, n^{\prime}-1}\right) .
$$

As usual the matrix elements between the zeroth level and the other levels should be treated separately. ${ }^{12}$ Corresponding to Eqs. (8) and (9) one arrives at

$$
P_{0 n^{\prime}}^{s s^{\prime}}=-i v_{F}^{2}\left|a_{s^{\prime} n^{\prime}}\right|^{2} \delta_{0, n^{\prime}-1}, \quad P_{n 0}^{s s^{\prime}}=i v_{F}^{2}\left|a_{s n}\right|^{2} \delta_{n-1,0} .
$$

It is easy to show that $\left|E_{s n}\right| \geqslant \Delta$ for all $n$, so one may omit the complex modulus signs in Eqs. (9) and (10). One now needs to sum the terms that include all combinations of the matrix elements, that is, $\sum^{++}, \sum^{+-}, \sum^{-+}$, and $\sum^{--}$. Here the superscript $+/-$ denotes the conduction/valence band. First, the levels $n>0$ are considered due to the peculiarity of the zeroth level. As shown in the appendix, the Hall conductivity $\sigma_{K}$ can be expressed as a sum of two terms, in one of which the prefactor is independent of $\Delta$ and a term in which it is linear in $\Delta, \sigma_{K}=\sigma_{K 0}+\delta \sigma_{K}$, with

$$
\sigma_{K 0}=\frac{g_{s} e^{2}}{h} \sum_{n=1}^{\infty}\left(n+\frac{1}{2}\right)\left(f_{n}^{+}-f_{n+1}^{+}+f_{n}^{-}-f_{n+1}^{-}\right)
$$

and

$$
\delta \sigma_{K}=\frac{g_{s} e^{2}}{2 h} \Delta \sum_{n=1}^{\infty}\left(\frac{f_{n}^{+}-f_{n}^{-}}{E_{n}}-\frac{f_{n+1}^{+}-f_{n+1}^{-}}{E_{n+1}}\right) .
$$


The factor $g_{s}$ is the spin degeneracy. The sums in Eqs. (11) and (12) go from $n=1$ to infinity because the zeroth level will be considered separately. At this point one should recall that these derivations are done for carriers in the vicinity of the $K$ point only. In order to take into account carriers near the $K^{\prime}$ point as well, the easiest way to do it is to replace $\Delta$ by $-\Delta$. Since the factor $\Delta$ appears in the first (odd) power in Eq. (12), the terms $\delta \sigma_{K}$ will cancel for $n>0$. For $n=0$ it can be proven that the contribution from the zeroth level is $\sigma_{y x}^{0, v}=g_{s} e^{2} / h f_{0}^{v}=$ $g_{s} e^{2} /(2 h)\left(f_{0}^{+, v}+f_{0}^{-, v}\right)$, with the notion that $f_{0}^{+, v}=f_{0}^{-, v}=$ $f_{0}^{v}$ for each valley $v$. One should not confuse the superscript \pm in $f_{0}^{ \pm, v}$ with the actual sign of the zeroth level energy in a particular valley. Thus, this term can easily be incorporated into the final expression for $\sigma_{y x}$, without any danger of double counting. Finally,

$$
\sigma_{y x}^{n d}=\frac{g_{s} e^{2}}{h} \sum_{n=0, v}^{\infty}\left(n+\frac{1}{2}\right)\left(f_{n}^{+, v}-f_{n+1}^{+, v}+f_{n}^{-, v}-f_{n+1}^{-, v}\right) .
$$

The summation over $v=1,-1$ is necessary since the zeroth levels of the $K$ and $K^{\prime}$ valleys do not coincide. The form of Eq. (13) is essentially the same as that of graphene on a $\mathrm{SiO}_{2}$ substrate ${ }^{12}$ since $g_{d}=2 g_{s}$ (the degeneracy with respect to the valley degree of freedom is $g_{v}=2$ ). Note that in Ref. 12 the overall degeneracy factor $g_{d}=4$ was denoted by the symbol $g_{s}$. At zero or very low temperatures and for the case of zero gap, Eq. (13) takes a simple form, ${ }^{12,16} \sigma_{y x}^{n d}= \pm 4 e^{2} / h(N+$ $1 / 2$ ), where $N$ is a non-negative integer. The last relation can be expressed in terms of the filling factor $v, \sigma_{y x}^{n d}=v e^{2} / h$, and, hence, ${ }^{17} v=4(N+1 / 2)$. The additional factor $1 / 2$ is the most obvious manifestation of the anomalous IQHE in gapless samples of monolayer graphene. The existence of a Landau-like level at zero energy, for $\Delta=0$, equally shared by the conduction and valence bands, is the key to understand the difference between the IQHE in graphene and in common semiconductors such as GaAs. ${ }^{18}$ A more detailed study ${ }^{19}$ has investigated the role of the symmetry of the disorder on the formation of a quantum Hall state in graphene. It is interesting to note that an expression similar to $\sigma_{K}=\sigma_{K 0}+\delta \sigma_{K}$ was derived in Ref. 8 for the valley Hall conductivity, at $B=0$, with the correction term proportional to $\Delta$ as in Eq. (12).

An equally important quantity for characterizing the magnetotransport properties of the materials is the longitudinal conductivity $\sigma_{x x}$. When a perpendicular magnetic field is applied, the diffusive contribution to the conductivity vanishes $\sigma_{x x}^{\text {dif }}=0$ and only the collisional (or hopping) contribution $\sigma_{x x}^{\text {col }}$ is important. The latter is given by ${ }^{13}$

$$
\sigma_{x x}^{\mathrm{col}}=\frac{\beta e^{2}}{2 S_{0}} \sum_{\zeta, \zeta^{\prime}} f\left(E_{\zeta}\right)\left[1-f\left(E_{\zeta^{\prime}}\right)\right] W_{\zeta \zeta^{\prime}}\left(y_{\zeta}-y_{\zeta^{\prime}}\right)^{2}
$$

where $W_{\zeta \zeta^{\prime}}$ is the transition rate between the states $|\zeta\rangle$ and $\left|\zeta^{\prime}\right\rangle$, while $y_{\zeta}=\langle\zeta|y| \zeta\rangle$ is the expectation value of the coordinate $y$. If scattering by impurities is assumed to be elastic, the transition rate $W_{\zeta \zeta^{\prime}}$ is given by

$$
W_{\zeta \zeta^{\prime}}=\frac{2 \pi n_{i}}{\hbar S_{0}} \sum_{\mathbf{q}}|U(\mathbf{q})|^{2}\left|F_{\zeta \zeta^{\prime}}(u)\right|^{2} \delta\left(E_{\zeta}-E_{\zeta^{\prime}}\right) \delta_{k_{x}, k_{x}^{\prime}+q_{x}},
$$

with $n_{i}$ the concentration of impurities and $\mathbf{q}$ the change in the electron wave vector. Further, $u=\ell_{c}^{2} q^{2} / 2, U(\mathbf{q})$ is the Fourier transform of the scattering potential, and $F_{\zeta \zeta^{\prime}}(u)$ is the form factor $F_{\zeta \zeta^{\prime}}(u)=\left\langle\zeta\left|e^{i \mathbf{q} \cdot \mathbf{r}}\right| \zeta^{\prime}\right\rangle$. The expectation value $y_{\zeta}$ is readily evaluated, and one finds $y_{\zeta}=\ell_{c}^{2} k_{x}$ and $y_{\zeta^{\prime}}=\ell_{c}^{2} k_{x}^{\prime}$. The impurity potential is taken to be that of screened charges $U(\mathbf{r})=\left(e^{2} / 4 \pi \varepsilon_{r} \varepsilon_{0} r\right) e^{-k_{s} r}$, where $k_{s}$ is the screening wave vector, $\varepsilon_{r}$ the relative permittivity, and $\varepsilon_{0}$ the permittivity of the vacuum. The Fourier transform of $U(\mathbf{r})$ is then given by $U(\mathbf{q})=U_{0} /\left(q^{2}+k_{s}^{2}\right)^{1 / 2}$, where $U_{0}=e^{2} /\left(2 \varepsilon_{r} \varepsilon_{0}\right)$. On inserting Eq. (15) in Eq. (14) one notices that the factor $\left(y_{\zeta}-y_{\zeta^{\prime}}\right)^{2}$ will not vanish even for elastic collisions $E_{\zeta}=E_{\zeta^{\prime}}$ due to the restriction $k_{x}=k_{x}^{\prime}+q_{x}$. This is possible because the Landau states are degenerate with respect to the wave vector $k_{x}$. Since the scattering is elastic and the eigenvalues do not depend on $k_{x}$, only the transitions $n \rightarrow n$ are allowed. The form factors then are given by

$$
\left|F_{n n}^{s s}(u)\right|^{2}=e^{-u}\left[a_{s n}^{2} L_{n-1}(u)+b_{s n}^{2} L_{n}(u)\right]^{2},
$$

for $n>0$ and $\left|F_{00}^{s s}(u)\right|^{2}=e^{-u}$ for $n=0$. This expression is identical to that of Ref. 20 in which, however, the gap has a different origin. Further, if the impurity potential is strongly short ranged (of the Dirac $\delta$-function type), one may use the approximation $k_{s} \gg q$. The factor $\left(y_{\zeta}-y_{\zeta^{\prime}}\right)^{2}$ is equal to $\ell_{c}^{4} q^{2} \cos ^{2} \varphi$. One should not forget the spin degree of freedom, denoted by $\sigma$, and it is here suppressed as a quantum number index. Transforming the sums to integrals, $\sum_{k_{x}, \sigma} \rightarrow g_{s} S_{0} / 2 \pi \ell_{c}^{2} \quad$ and $\quad \sum_{\mathbf{q}} \rightarrow S_{0} /\left(2 \pi \ell_{c}\right)^{2} \int d u \int d \varphi$, and using polar coordinates, Eq. (14) takes the form

$$
\sigma_{x x}^{\mathrm{col}}=\frac{g_{s} e^{2}}{h} \frac{\beta n_{i} U_{0}^{2}}{4 u_{c s} \hbar \omega_{c}} \sum_{s, n, v} I_{s n} f\left(E_{s n}^{v}\right)\left[1-f\left(E_{s n}^{v}\right)\right],
$$

where $u_{c s}=\ell_{c}^{2} k_{s}^{2} / 2$ and

$$
I_{s n}=\int_{0}^{\infty} u\left|F_{n n}^{s s}(u)\right|^{2} d u .
$$

The summation over $k_{x}^{\prime}$ does not produce any additional factor due to the Kronecker symbol $\delta_{k_{x}, k_{x}^{\prime}+q_{x}}$. In Eq. (17) the summation over the two valleys, i.e., over $v$, is performed, and for that reason only $g_{s}$, instead of $g_{d}$, appears as the degeneracy factor. Similarly to the case of the Hall conductivity, this summation is necessary because the energy spectra in the two valleys differ in the zeroth level. The integration in Eq. (18) is carried out analytically using the orthogonality of the polynomials $L_{n}(u)$ and their recurrence relation $(n+1) L_{n+1}(u)-(2 n+1-u) L_{n}(u)+n L_{n-1}(u)=0$. The result is

$I_{s n}=(2 n+1)\left|b_{s n}\right|^{4}-2 n\left|a_{s n}\right|^{2}\left|b_{s n}\right|^{2}+(2 n-1)\left|a_{s n}\right|^{4}$.

When $\Delta=0$, the expression for $I_{s n}$ reduces to $2 n / 4$, which means that the minima of $\sigma_{x x}$ occur at odd filling factors $v=2 n+1$ in accord with Ref. 16. Recall that in common semiconductors the minima occur at even filling factors $v=2 n$ and that $\sigma_{x x} \propto(2 n+1)$.

Scattering by impurities also leads to a $k_{x}$-dependent shift or energy correction $\Delta \bar{E}_{s n}=\left\langle s n k_{x}|U(\mathbf{r})| s n k_{x}\right\rangle$ of the eigenvalues that has already been evaluated in Ref. 12 . With 
$\xi=k_{x} \ell_{c}$ and $C=V_{0} / \ell_{c} L_{y}$ the result for $n>0$ is

$\Delta \bar{E}_{s n}=\frac{s C e^{-\xi^{2}}}{2^{n+1} n ! \sqrt{\pi}}\left[\left|b_{s n}\right|^{2} H_{n}^{2}(\xi)+2 n\left|a_{s n}\right|^{2} H_{n-1}^{2}(\xi)\right]$,

while for $n=0$ it is $\Delta \bar{E}_{s 0}=s C e^{-\xi^{2}} / \sqrt{\pi}$. However, it is difficult to assess the quantity $C$ using its formal dependence on the parameters of the material. A convenient approach is phenomenological and assumes that $C$ is close ${ }^{12}$ to the value of the broadening $\Gamma$. This can be partly justified by the fact that both quantities are directly related to the scattering by impurities and by the fact that both have the same dependence on magnetic field $\sqrt{B}$. Further, magnetotransport studies ${ }^{21}$ of the activation gap may explain the origin of a particular splitting: for instance, $E(v= \pm 1)$ is expected ${ }^{22}$ to be on the scale of $e^{2} / \varepsilon \ell_{c}$, hence proportional to $\sqrt{B}$, in accordance with the previous assumption $C \approx \Gamma$. As in Ref. 12 the value of $k_{x}$ is taken to be $10^{6} \mathrm{~m}^{-1}$.

\section{NUMERICAL RESULTS}

First, the Hall conductivity $\sigma_{y x}$ vs. the magnetic field is shown in Fig. 2 for fixed electron concentration $n_{e}=$ $10^{12} \mathrm{~cm}^{-2}$. The solid curve corresponds to the gapped graphene while the dotted one to the usual, gapless monolayer graphene on $\mathrm{SiO}_{2}$ substrate. The difference between the two curves is most pronounced for high magnetic fields in line with the spectrum given by Eq. (6).

In Fig. 3 the Hall conductivities of graphene on a h-BN substrate and on a $\mathrm{SiO}_{2}$ substrate are shown as functions of the electron concentration $n_{e}$ for a fixed magnetic field $B=14 \mathrm{~T}$. The value of the band gap is estimated to be $2 \Delta=53 \mathrm{meV}$ from $a b$ initio calculations. ${ }^{3}$ However, we use the value $\Delta=53 \mathrm{meV}$ for illustrative purposes. The electron concentration can be tuned by applying a gate. ${ }^{10}$ The common case $\Delta=0$ is given by the dotted red curve and exhibits the usual behavior. The correction $\Delta \bar{E}_{s n}$ to the energy levels due to impurities is not included. Notice the

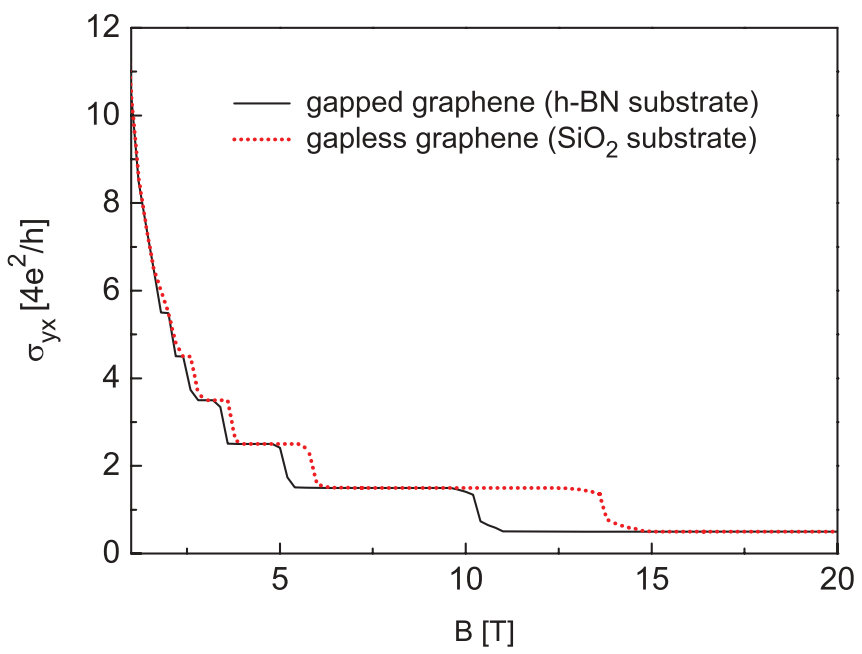

FIG. 2. (Color online) The Hall conductivity $\sigma_{y x}$ vs. the magnetic field at fixed electron concentration $n_{e}=10^{12} \mathrm{~cm}^{-2}$. The solid curve is for a gapped monolayer graphene $(\Delta=53 \mathrm{meV})$ while the dotted one for graphene on $\mathrm{SiO}_{2}$ substrate.

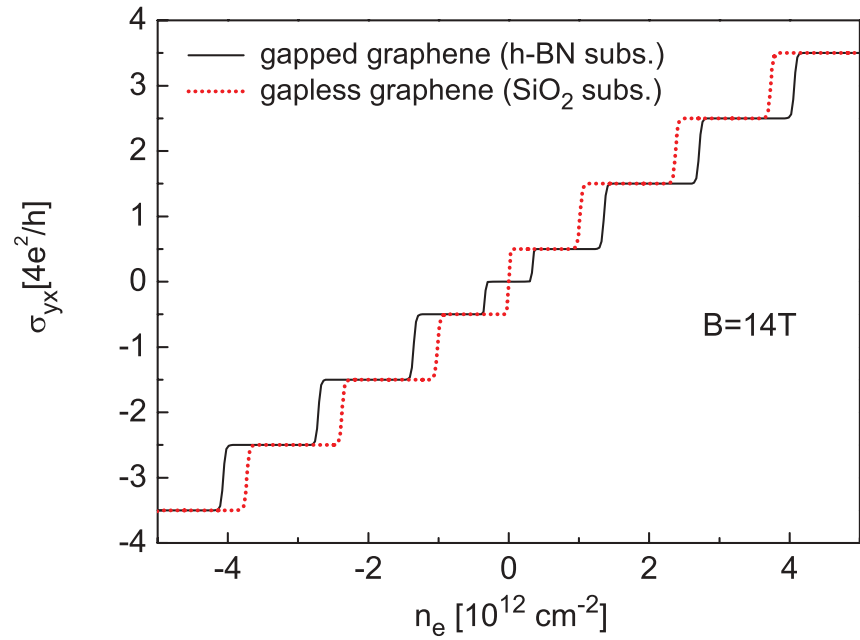

FIG. 3. (Color online) The Hall conductivity $\sigma_{y x}$ vs. the electron concentration $n_{e}$ for a monolayer graphene on a h-BN substrate with the mass term $\Delta=53 \mathrm{meV}$. The dotted red curve corresponds to graphene supported by a $\mathrm{SiO}_{2}$ substrate.

difference of the two curves in the vicinity of $n_{e}=0$. This is in line with experimental observations ${ }^{1}$ that additional plateaus derived from $n=0$ are already visible at considerably smaller magnetic fields $(B>8.5 \mathrm{~T})$ than reported for monolayer graphene ${ }^{11}$ on $\mathrm{SiO}_{2}$ substrates. Further insight into the problem can be gained by inspecting the Hall conductivity near the Dirac point but at different values of the magnetic field. In Fig. 4 the value of $\sigma_{x y}\left(=-\sigma_{y x}\right)$ is plotted vs. the applied voltage $V_{g}^{0}$ for three magnetic fields: $8 \mathrm{~T}$ (black, dashed curve), $11 \mathrm{~T}$ (red, dotted curve), and $14 \mathrm{~T}$ (blue, dash-dotted curve). For comparison, it is also plotted in the inset, for $\Delta=0$, at the same magnetic fields. The relation between the voltage $V_{g}^{0}$ and the electron concentration $n_{e}$ is linear ${ }^{23} n_{e}=\varepsilon \varepsilon_{0} /(t e) V_{g}^{0}$, where $t$ is the thickness of the substrate, $\varepsilon$ and $\varepsilon_{0}$ are the (relative) permittivity of the substrate and the vacuum, respectively. The other parameters are the same as those for Fig. 3.

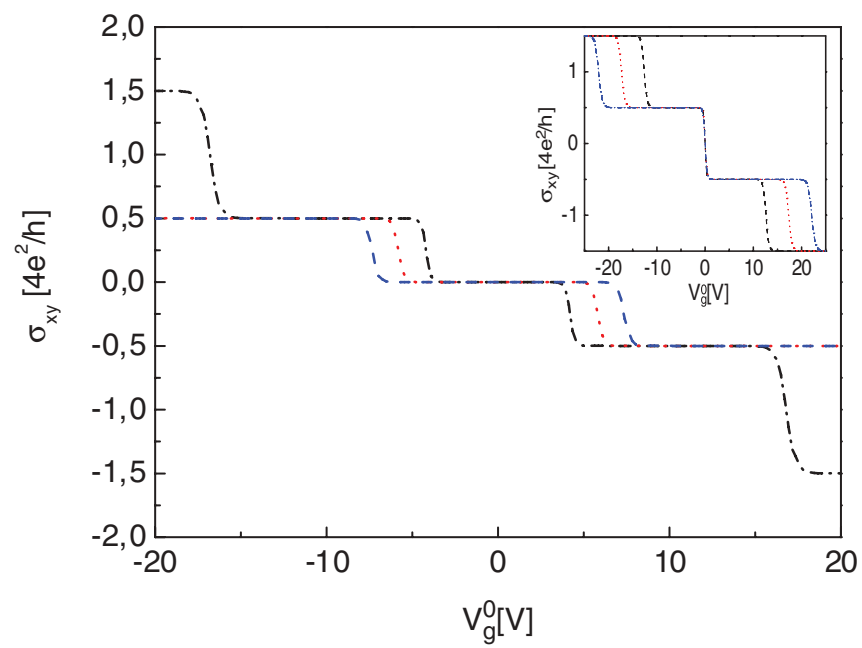

FIG. 4. (Color online) The Hall conductivity $\sigma_{x y}$ vs. the applied voltage $V_{g}^{0}$ for three different magnetic fields: $8 \mathrm{~T}$ (black dashed curve), $11 \mathrm{~T}$ (red dotted curve), and $14 \mathrm{~T}$ (blue dash-dotted curve), all for $\Delta=53 \mathrm{meV}$. The inset shows $\sigma_{x y}$ for $\Delta=0$. 

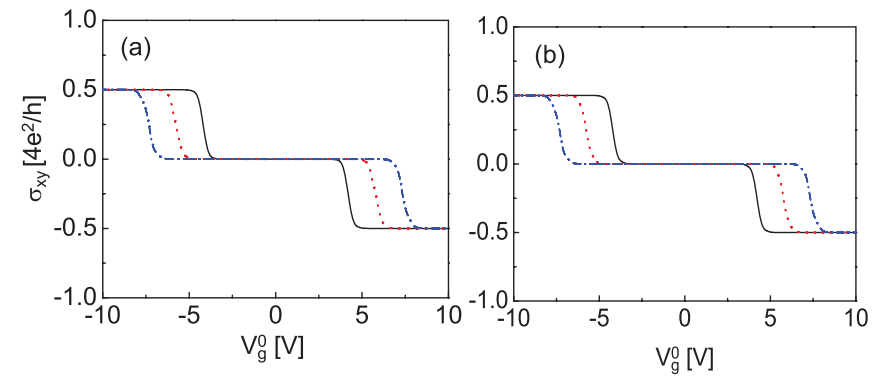

FIG. 5. (Color online) The Hall conductivity $\sigma_{x y}$ vs. the voltage $V_{g}^{0}$ for three values of magnetic field: $8 \mathrm{~T}$ (black dashed curve), $11 \mathrm{~T}$ (red dotted curve), and $14 \mathrm{~T}$ (blue dash-dotted curve). In (a) the correction to the eigenvalues due to impurities is neglected, while in (b) it is included.

As can be seen by contrasting the inset with the main figure, the zero-energy level is replaced by two levels, one for each valley. In general, the higher the magnetic field the higher $V_{g}^{0}$ is needed to fill the next Landau level. This results from the fact the density of states per spin is $e B / h$. For clarity, Fig. 5 shows the Hall conductivity versus $V_{g}^{0}$ in the vicinity of the Dirac point for three different magnetic fields, $8 \mathrm{~T}, 11 \mathrm{~T}$, and $14 \mathrm{~T}$ (as in the previous case). In Fig. 5(a) the energy correction due to impurities is neglected, while in Fig. 5(b) it is included. As one can see, the difference is minimal and not visible on the scale used. This is because the mass term $\Delta \approx 53 \mathrm{meV}$ is much larger than this correction $\Delta E_{0} \approx 11 \mathrm{meV}$ (for the zeroth level).

Experimentally, one measures the longitudinal resistivity $\rho_{x x}$ related to the longitudinal conductivity $\sigma_{x x}$ by

$$
\rho_{x x}=\frac{\sigma_{x x}}{\sigma_{x x}^{2}+\sigma_{y x}^{2}} .
$$

Of course, $\sigma_{x x}=\sigma_{x x}^{\text {col }}$, since the diffusive component vanishes in a perpendicular magnetic field. Figure 6 shows the longitudinal resistivity $\rho_{x x}$ as a function of the electron

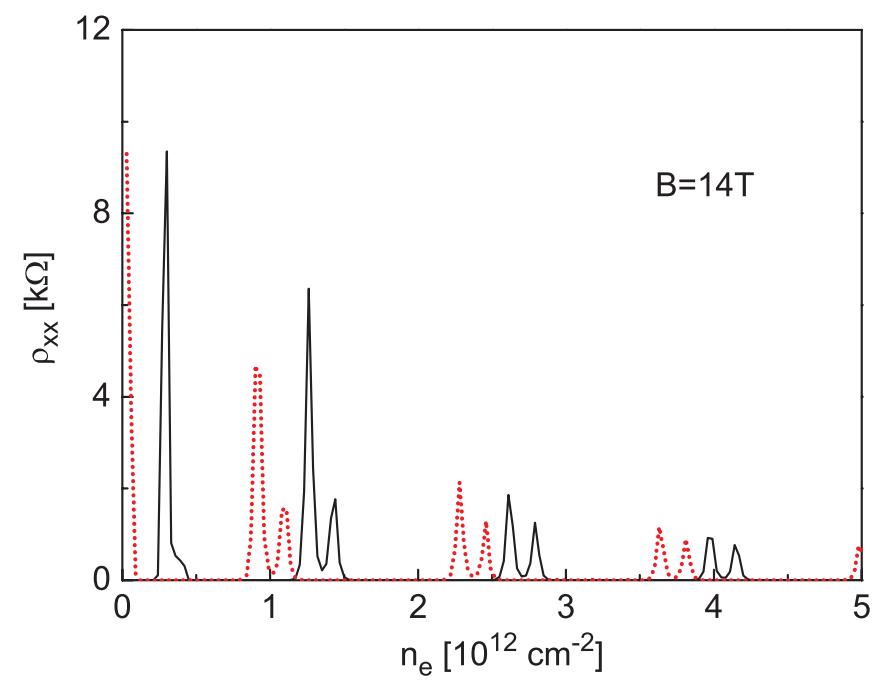

FIG. 6. (Color online) The longitudinal resistivity $\rho_{x x}$ vs. the electron concentration $n_{e}$ at fixed magnetic field $B=14 \mathrm{~T}$. The dotted red curve corresponds to graphene on a $\mathrm{SiO}_{2}$ substrate. The other parameters are $\Delta=53 \mathrm{meV}$ and $T=4.2 \mathrm{~K}$.

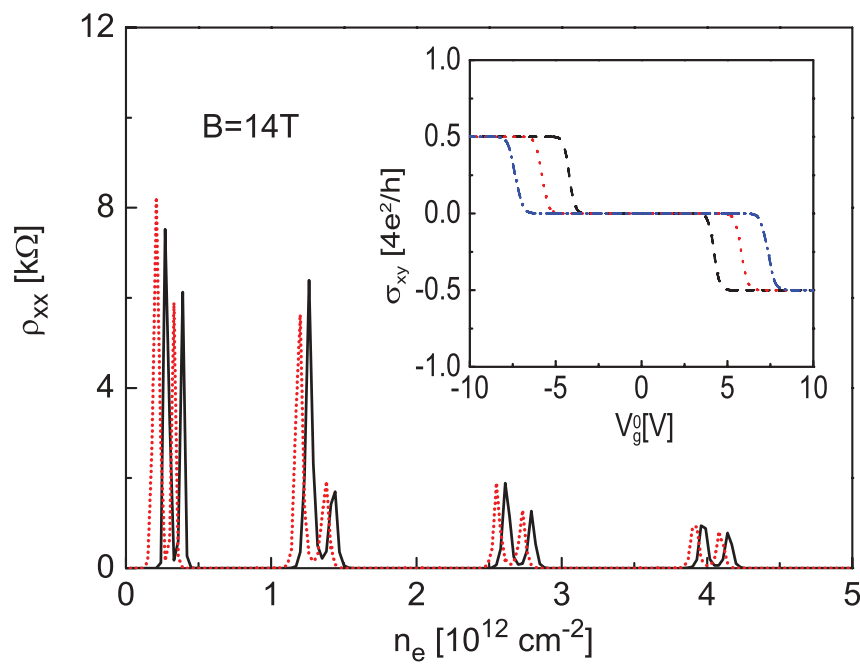

FIG. 7. (Color online) As in Fig. 6 but with the correction $\Delta \bar{E}_{s n}$ to the energy levels included. The magnetic field is $B=14 \mathrm{~T}$. The dotted (red) curve corresponds to graphene supported by a $\mathrm{SiO}_{2}$ substrate. The inset shows the conductivity $\sigma_{y x}$, with correction to the energy levels, for three different magnetic fields (the same as those in Fig. 4). The other parameters are $\Delta=53 \mathrm{meV}, A=50$, $n_{i}=1.3 \times 10^{9} \mathrm{~cm}^{-2}$, and $T=4.2 \mathrm{~K}$.

concentration $n_{e}$ at fixed magnetic field $B=14 \mathrm{~T}$. The solid curve corresponds to graphene on a h-BN substrate and the dotted one to graphene on $\mathrm{SiO}_{2}$ substrate. The difference between the two curves is most visible near zero concentration. This is expected since the energy spectra differ the most near the Dirac point, where one has two levels, $-\Delta$ and $+\Delta$, in place of a single one for $\Delta=0$.

Figure 7 shows the resistivity $\rho_{x x}$ versus the electron concentration $n_{e}$ with the correction $\Delta \bar{E}_{s n}$ to the energy levels (due to impurities) taken into account. The dimensionless parameter $A$ was taken to be $A=50$, the impurity concentration $n_{i}=1.3 \times 10^{9} \mathrm{~cm}^{-2}$, and the temperature $T=4.2 \mathrm{~K}$. Recall that the phenomenological parameter $A$ depends on the concentration $n_{i}$ and takes on larger values for cleaner samples. The values of $\rho$ are smaller due to the sensitivity of the Fermi factors $f\left(E_{s n}\right)$ on the position of the levels. The inset shows the Hall conductivity versus $n_{e}$ for three different magnetic fields, the same as those in Fig. 4. By contrasting the dashed red curves in Figs. 6 and 7, one sees no gap in Fig. 6, in agreement with the observed maxima in $\rho_{x x}$ for $n_{e}=0$, whereas there is one in Fig. 7 due to the energy correction; this agrees with the results of Ref. 12.

Finally, in order to better understand the nature of the double peaks of $\rho_{x x}$ in Figs. 6 and 7, we plot $\rho_{x x}, \sigma_{x x}$, and $\sigma_{y x}$ in Fig. 8 as functions of the electron concentration $n_{e}$ for $B=14 \mathrm{~T}$ but when $\Delta \bar{E}_{s n}=0$. The values of $\rho_{x x}$ (black, solid curve) and $\sigma_{x x}$ (red, dotted curve) are given on the left $y$ axis and those of $\sigma_{y x}$ on the right axis. The other parameters are the same as in Fig. 6. Note that the minima in $\rho_{x x}$ correspond to plateaus of $\sigma_{y x}$ as it should be. It can be seen from the figure that the double peaks in $\rho_{x x}\left(n_{e}\right)$ are a consequence of a relatively wide transitional region between two plateaus in $\sigma_{y x}$. Experimentally, double peaks are indeed observed in graphene on a h-BN substrate ${ }^{24}$ and referred to as a gap collapse at odd fillings. This behavior 


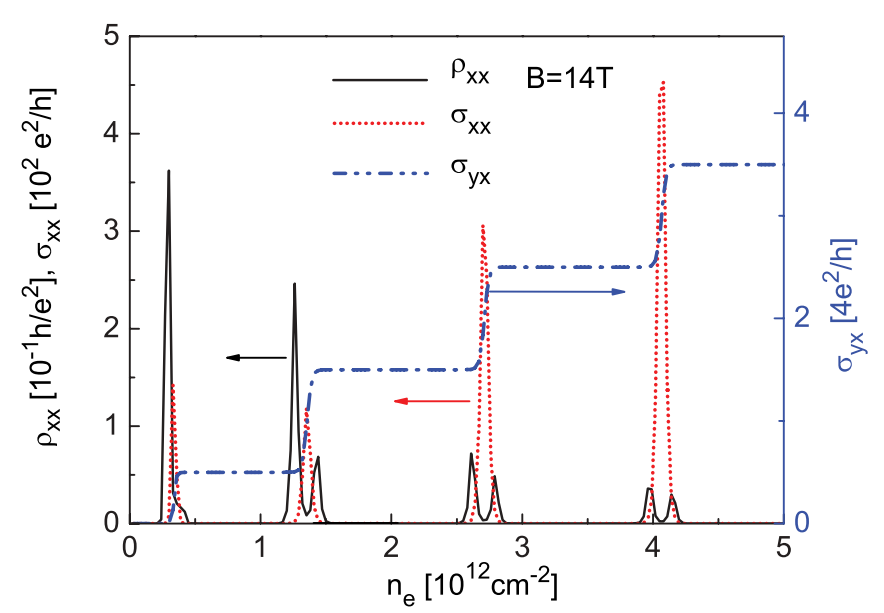

FIG. 8. (Color online) (Left axis) Longitudinal resistivity $\rho_{x x}$ (solid black curve) and conductivity $\sigma_{x x}$ (dotted red curve) vs. the electron density $n_{e}$. (Right axis) The Hall conductivity $\sigma_{y x}$ (dash-dotted blue curve) vs. $n_{e}$.

tends to appear in cleaner samples and disappears on sample contamination. $^{24}$

\section{CONCLUDING REMARKS}

We investigated the magnetotransport properties of graphene placed on a boron-nitride substrate and derived analytical expressions for the Hall conductivity $\sigma_{y x}$ and the longitudinal resistivity $\rho_{x x}$ under certain assumptions regarding the scattering potential. It was shown that the analytical form of $\sigma_{y x}$ is essentially the same as that for the usual, gapless graphene samples, provided the sum (over states) is carried out separately for the $K$ and $K^{\prime}$ valleys. This results in the fact that the mass term $\Delta$, in the prefactor of one contribution to $\sigma_{y x}$, is canceled in the final sum over the two valleys.

The results are also in line with the theoretical findings of Ref. 5 that the usual IQHE is not seen in a single valley but is restored in the total Hall conductivity. A new plateau near the Dirac point is predicted to appear as a consequence of the existence of the band gap. The width of the plateau increases with the mass term when the Fermi level is varied at fixed $B$. The above analysis is valid under the assumption that the mass term $\Delta$ is not too large compared to the first Landau level $\hbar \omega_{c}$ which is fulfilled for a h-BN substrate and not too high magnetic fields. However, for the case of Si-C substrate, with ${ }^{25} 2 \Delta=260 \mathrm{meV}$, this requirement can hardly be met and suppression of Hall plateaus is predicted, also in accord with Ref. 5. Further, the scattering by impurities is taken into account. It induces a plateau near $n_{e}=0$ in gapless, monolayer graphene and very slightly widens that in gapped graphene. This is also in agreement with previous numerical studies $^{26}$ which considered electron-electron interaction as a source of lifting the degeneracy of the levels with respect to $k_{x}$. The numerical results for $\sigma_{y x}$ show that plateaus are further shifted (horizontally) to the right due to the band gap $2 \Delta$.

As far as the resistivity $\rho_{x x}$ is concerned, we demonstrated that it exhibits an oscillatory behavior vs. the electron concentration $n_{e}$ as expected. The peaks are shifted by both scattering by impurities and the band gap induced by the substrate. The values of $\rho_{x x}$, when the correction to the eigenvalues is included, are somewhat smaller due to sensitivity of the Fermi factors on the positions of the energy levels. At the end we addressed the problem of double peaks in $\rho_{x x}\left(n_{e}\right)$, which have been observed in recent experimental data. ${ }^{24}$ Within the framework of the model presented here, the reason for their onset is attributed to the wide transitional regions between two adjacent plateaus in the Hall conductivity. The latter in turn results from the motion of the Fermi level through the nonequidistant energy levels in contrast with the equidistant ones in common semiconductors. However, there could be additional physical reasons for the onset of the double peaks which may even enlarge gaps.

\section{ACKNOWLEDGMENTS}

We thank M. Zarenia for a critical reading of the manuscript and useful suggestions. The work of P.K. was supported by the Serbian Ministry of Education and Science, within the Project No. TR 32008, and that of P.V. by the Canadian NSERC Grant No. OGP0121756.

\section{APPENDIX: DERIVATION OF THE HALL CONDUCTIVITY}

Starting with Eq. (7), for $\Gamma_{\zeta}=0$, and setting $C_{n n^{\prime}}^{s s^{\prime}}=$ $\left|a_{s n} b_{s^{\prime} n^{\prime}}\right|^{2}$ and $A=-\hbar e^{2} v_{F}^{2} / S_{0}$ for brevity, leads to

$\sigma_{y x}^{n d}=A \sum_{k n n^{\prime} s s^{\prime}} \frac{\left(f_{n k}^{s}-f_{n^{\prime} k}^{s^{\prime}}\right)\left[C_{n n^{\prime}}^{s s^{\prime}} \delta_{n^{\prime}, n-1}-C_{n^{\prime} n}^{s^{\prime} s} \delta_{n^{\prime}, n+1}\right]}{\left(E_{s n}-E_{s^{\prime} n^{\prime}}\right)^{2}}$,

where $k=k_{x}$. Due to the Kronecker $\delta$ symbols the sums start at different values so one can split the two terms

$$
\begin{aligned}
\sigma_{y x}^{n d}= & A \sum_{k, n=2, s s^{\prime}} \frac{f_{n}^{s}-f_{n-1}^{s^{\prime}}}{\left(E_{s n}-E_{s^{\prime} n-1}\right)^{2}} C_{n, n-1}^{s s^{\prime}} \\
& -A \sum_{k, n=1, s s^{\prime}} \frac{f_{n}^{s}-f_{n+1}^{s^{\prime}}}{\left(E_{s n}-E_{s^{\prime} n+1}\right)^{2}} C_{n+1, n}^{s^{\prime} s} .
\end{aligned}
$$

Consider first the sum for $s=+$ and $s^{\prime}=+$, denoted by $\sum^{++}$. On making the changes $n-1 \rightarrow m \rightarrow n$ in the first sum, one may combine the two sums into one,

$$
\sum^{++}=2 A \sum_{k, n=1} \frac{f_{n+1}^{+}-f_{n}^{+}}{\left(E_{n}-E_{n+1}\right)^{2}} C_{n+1, n}^{++},
$$

where $E_{s n}=s E_{n}\left(E_{n}>0\right)$ and

$$
C_{n+1, n}^{++}=\frac{E_{n+1}+\Delta}{2 E_{n+1}} \frac{E_{n}-\Delta}{2 E_{n}}=\frac{D+E}{4 E_{n} E_{n+1}},
$$

with $D=E_{n} E_{n+1}-\Delta^{2}$ and $E=\left(E_{n}-E_{n+1}\right) \Delta$.

In like manner, one evaluates the sum $\sum^{--}$

$$
\sum^{--}=2 A \sum_{k, n=1} \frac{f_{n+1}^{-}-f_{n}^{-}}{\left(E_{n}-E_{n+1}\right)^{2}} C_{n+1, n}^{--},
$$


with

$$
C_{n+1, n}^{--}=(D-E) / 4 E_{n} E_{n+1} .
$$

On the other hand, for $\sum^{+-}$the order in which indices + and - appear is important. Hence, one obtains

$$
\begin{aligned}
\sum^{+-}= & A \sum_{k, n=1}\left[\frac{f_{n+1}^{+}-f_{n}^{-}}{\left(E_{-, n+1}-E_{+, n}\right)^{2}} C_{n+1, n}^{+-}\right. \\
& \left.-\frac{f_{n}^{+}-f_{n+1}^{-}}{\left(E_{+, n}-E_{-, n+1}\right)^{2}} C_{n+1, n}^{-+}\right],
\end{aligned}
$$

with

$$
C_{n+1, n}^{+-}=(B+C) / 4 E_{n} E_{n+1},
$$

while $B=E_{n} E_{n+1}+\Delta^{2}$ and $C=\left(E_{n}+E_{n+1}\right) \Delta$.

Similarly, the expression for $\sum^{-+}$is

$$
\begin{aligned}
\sum^{-+}= & A \sum_{k, n=1}\left[\frac{f_{n+1}^{-}-f_{n}^{+}}{\left(E_{+, n+1}-E_{-, n}\right)^{2}} C_{n+1, n}^{-+}\right. \\
& \left.-\frac{f_{n}^{-}-f_{n+1}^{+}}{\left(E_{-, n}-E_{+, n+1}\right)^{2}} C_{n+1, n}^{+-}\right],
\end{aligned}
$$

with

$$
C_{n+1, n}^{-+}=(B-C) / 4 E_{n} E_{n+1} .
$$

The denominators in Eqs. (A7) and (A9) are equal to $\left(E_{n}+\right.$ $\left.E_{n+1}\right)^{2}$ due to the squaring. Adding Eqs. (A3) and (A5) gives $S_{1}=\sum^{++}+\sum^{--}$in the form

$$
\begin{aligned}
S_{1}= & 2 A \sum_{k, n=1}\left[\frac{\left[f_{n+1}^{+}-f_{n}^{+}+f_{n+1}^{-}-f_{n}^{-}\right]}{4 E_{n} E_{n+1}\left(E_{n}-E_{n+1}\right)^{2}} D\right. \\
& \left.+\frac{f_{n+1}^{+}-f_{n}^{+}-\left(f_{n+1}^{-}-f_{n}^{-}\right)}{4 E_{n} E_{n+1}\left(E_{n}-E_{n+1}\right)^{2}} E\right] .
\end{aligned}
$$

By setting $S_{2}=\sum^{+-}+\sum^{-+}$one obtains

$$
\begin{aligned}
S_{2}= & 2 A \sum_{k, n=1}\left[\frac{f_{n+1}^{+}-f_{n}^{+}+f_{n+1}^{-}-f_{n}^{-}}{4 E_{n} E_{n+1}\left(E_{n}+E_{n+1}\right)^{2}} B\right. \\
& \left.+\frac{f_{n+1}^{+}-f_{n}^{-}-\left(f_{n+1}^{-}-f_{n}^{+}\right)}{4 E_{n} E_{n+1}\left(E_{n}+E_{n+1}\right)^{2}} C\right] .
\end{aligned}
$$

Now, Eqs. (A11) and (A12) should be added using the expressions for $B, C, D$, and $E$. For the terms $\propto E_{n} E_{n+1}$ in $B$ and $D$ one arrives at $\left(a=\hbar^{2} \omega_{c}^{2}\right)$

$$
\frac{1}{\left(E_{n}+E_{n+1}\right)^{2}}+\frac{1}{\left(E_{n}-E_{n+1}\right)^{2}}=\frac{4 \Delta^{2}+2(2 n+1) a}{a^{2}},
$$

while the difference of the two fractions on the left side is equal to $-4 E_{n} E_{n+1} / a^{2}$. Adding the terms $\propto B, D$ gives

$$
(A / a) \sum_{k, n=1}\left[f_{n+1}^{+}-f_{n}^{+}+f_{n+1}^{-}-f_{n}^{-}\right](2 n+1),
$$

which leads to Eq. (11) after summation over $k=k_{x}\left(\sum_{k_{x}, \sigma} \rightarrow\right.$ $g_{s} S_{0} / 2 \pi \ell_{c}^{2}$ ). We consider only terms up to the first power in $\Delta$. On the other hand, the terms $\propto E, C$ give

$$
\begin{aligned}
\sum_{k, n=1} & \frac{2 A \Delta}{4 E_{n} E_{n+1}}\left[\frac{f_{n+1}^{+}-f_{n}^{+}-f_{n+1}^{-}+f_{n}^{-}}{E_{n}+E_{n+1}}\right. \\
& \left.+\frac{f_{n+1}^{+}-f_{n}^{-}-f_{n+1}^{-}+f_{n}^{+}}{E_{n}-E_{n+1}}\right] \\
= & -\frac{A}{a} \sum_{k, n=1} \frac{\Delta}{E_{n} E_{n+1}}\left[E_{n}\left(f_{n+1}^{+}-f_{n+1}^{-}\right)-E_{n+1}\left(f_{n}^{+}-f_{n}^{-}\right)\right] \\
= & -\frac{A}{a} \sum_{k, n=1} \Delta\left[\frac{f_{n+1}^{+}-f_{n+1}^{-}}{E_{n+1}}-\frac{f_{n}^{+}-f_{n}^{-}}{E_{n}}\right] . \quad \text { (A15) }
\end{aligned}
$$

This leads to Eq. (12) after summation over $k=k_{x}$. The derivation given above is valid for $n \geqslant 1$. It can be extended for $n=0$; see the text above Eq. (13).
${ }^{1}$ C. R. Dean, A. F. Young, I. Meric, C. Lee, L. Wang, S. Sorgenfrei, K. Watanabe, T. Taniguchi, P. Kim, K. L. Shepard, and J. Hone, Nat. Nanotechnol. 5, 722 (2010).

${ }^{2}$ W. Gannett, W. Regan, K. Watanabe, T. Taniguchi, M. F. Crommie, and A. Zettl, Appl. Phys. Lett. 98, 242105 (2011).

${ }^{3}$ G. Giovannetti, P. A. Khomyakov, G. Brocks, P. J. Kelly, and J. van den Brink, Phys. Rev. B 76, 073103 (2007).

${ }^{4}$ J. Jung, Z. Qiao, Q. Niu, and A. H. MacDonald, Nanoletters 12, 2936 (2012).

${ }^{5}$ L. Jiang, Y. Zheng, H. Li, and H. Shen, Nanotechnology 21, 145703 (2010).

${ }^{6}$ G. W. Semenoff, V. Semenoff, and F. Zhou, Phys. Rev. Lett. 101, 087204 (2008).

${ }^{7}$ L. Ci, L. Song, C. Jin, D. Jariwala, D. Wu, Y. Li, A. Srivastava, Z. F. Wang, K. Storr, L. Balicas, F. Liu, and P. M. Ajayan, Nat. Mater. 9, 430 (2010).
${ }^{8}$ D. Xiao, W. Yao, and Q. Niu, Phys. Rev. Lett. 99, 236809 (2007).

${ }^{9}$ Z. Qiao, S. A. Yang, B. Wang, Y. Yao, and Q. Niu, Phys. Rev. B 84, 035431 (2011)

${ }^{10}$ A. H. Castro Neto, F. Guinea, N. M. R. Peres, K. S. Novoselov, and A. K. Geim, Rev. Mod. Phys. 81, 109 (2009).

${ }^{11}$ Y. Zhang, Z. Jiang, J. P. Small, M. S. Purewal, Y.-W. Tan, M. Fazlollahi, J. D. Chudow, J. A. Jaszczak, H. L. Stormer, and P. Kim, Phys. Rev. Lett. 96, 136806 (2006).

${ }^{12}$ P. M. Krstajić and P. Vasilopoulos, Phys. Rev. B 83, 075427 (2011). ${ }^{13}$ M. Charbonneau, K. M. Van Vliet, and P. Vasilopoulos, J. Math. Phys. 23, 318 (1982); P. Vasilopoulos, Phys. Rev. B 32, 771 (1985).

${ }^{14}$ Y. Zheng and T. Ando, Phys. Rev. B 65, 245420 (2002).

${ }^{15}$ M. E. Raikh and T. V. Shahbazyan, Phys. Rev. B 47, 1522 (1993).

${ }^{16}$ V. P. Gusynin and S. G. Sharapov, Phys. Rev. Lett. 95, 146801 (2005). 
${ }^{17}$ I. F. Herbut, Phys. Rev. B 75, 165411 (2007).

${ }^{18}$ A. K. Geim and K. S. Novoselov, Nat. Mater. 6, 183 (2007).

${ }^{19}$ P. M. Ostrovsky, I. V. Gornyi, and A. D. Mirlin, Phys. Rev. B 77, 195430 (2008).

${ }^{20}$ M. Tahir and K. Sabeeh, J. Phys.: Condens. Matter 24, 135005 (2012).

${ }^{21}$ A. J. M. Giesbers, L. A. Ponomarenko, K. S. Novoselov, A. K. Geim, M. I. Katsnelson, J. C. Maan, and U. Zeitler, Phys. Rev. B 80, 201403(R) (2009).

${ }^{22}$ Z. Jiang, Y. Zhang, H. L. Stormer, and P. Kim, Phys. Rev. Lett. 99, 106802 (2007).
${ }^{23}$ K. S. Novoselov, A. K. Geim, S. V. Morozov, D. Jiang, M. I. Katsnelson, I. V. Grigorieva, S. V. Dubonos, and A. A. Firsov, Nature (London) 438, 197 (2005).

${ }^{24}$ A. F. Young, C. R. Dean, L. Wang, H. Ren, P. Cadden-Zimansky, K. Watanabe, T. Taniguchi, J. Hone, K. L. Shepard, and P. Kim, Nat. Phys. 8, 550 (2012).

${ }^{25}$ S. Y. Zhou, G.-H. Gweon, A. V. Fedorov, P. N. First, W. A. de Heer, D.-H. Lee, F. Guinea, A. H. Castro Neto, and A. Lanzara, Nat. Mater. 6, 770 (2007).

${ }^{26}$ Y. Barlas, R. Côté, K. Nomura, and A. H. MacDonald, Phys. Rev. Lett. 101, 097601 (2008). 